\title{
Cystic Lateral Neck Lesions: Etiologic and Differential Diagnostic Significance in a Series of 133 Patients
}

\author{
ACHIM FRANZEN ${ }^{1}$, THOMAS GÜNZEL ${ }^{2}$, ANDRE BUCHALI ${ }^{3}$ and ANNEKATRIN COORDES ${ }^{4}$ \\ ${ }^{1}$ Department of Otorhinolaryngology, Head and Neck Surgery, \\ Brandenburg Medical School Theodor Fontane, Ruppiner University Hospital, Neuruppin, Germany; \\ ${ }^{2}$ Department of Otorhinolaryngology and Head and Neck Surgery, Borromaeus-Hospital, Leer, Germany; \\ ${ }^{3}$ Department of Radio-oncology, Brandenburg Medical School Theodor Fontane, \\ Ruppiner University Hospital, Neuruppin, Germany; \\ ${ }^{4}$ Department of Otorhinolaryngology, Head and Neck Surgery, \\ Charite Medical School Berlin, Virchow Hospital, Berlin, Germany
}

\begin{abstract}
Background: Patients presenting with a cystic lateral neck lesion may present diagnostic challenges against a backdrop of varied non-malignant and malignant etiologies. Patients: A total of 133 consecutive cases who underwent cystic neck tumor removal were evaluated for etiology and diagnostic procedure in order to develop an algorithm for therapeutic efficiency. Results: In 92 of 133 cases, a non-malignant tumor was diagnosed. In 41 cases, malignant lymphadenopathy was found. In cases with malignancy, males $(p=0.001)$ and the elderly $(p=0.001)$ were affected more frequently. Primary tumors were discovered by pan-endoscopy before neck surgery or in a second panendoscopy (with tonsillectomy and mapping biopsies) in cases with histologically confirmed squamous cell carcinoma. During intraoperative frozen-section evaluation (40 cases), a total of 30 patients underwent neck dissection during the first neck operation. Conclusion: In patients older than 40 years who present with cystic neck lesions, we recommend pan-endoscopy and intraoperative frozen section in cases where malignancy is suspected in order to avoid secondary neck dissections and delays in therapy.
\end{abstract}

A cystic lesion of the lateral neck is a relatively rare occurrence for an Ear, Nose and Throat (ENT) specialist. The majority of cases are interpreted as branchial cleft cysts that

Correspondence to: Annekatrin Coordes, MD, Ph.D., Department of Otorhinolaryngology, Head and Neck Surgery, Charité University Hospital, Campus Virchow Klinikum, Augustenburger Platz 1, 13353 Berlin, Germany. Tel: +49 30450555602, Fax: +49 30450555970, e-mail: annekatrin.coordes@ charite.de

Key Words: Cystic cervical mass, lateral neck, differential diagnosis, pan-endoscopy, frozen section evaluation. arise from the second branchial arch and become symptomatic in children and younger adults (1). On the other hand, there are a wide variety of neck mass etiologies including congenital, local or systemic inflammatory, benign and malignant causes (2). Cystic metastases may represent a unique entity with different etiological features such as a lack of exposure to risk factors and presentation in younger or female patients. Patient age may be an independent predictive factor for malignancy $(2,3)$. In this way, a cystic neck lesion may represent a cystic metastasis of squamous cell carcinoma (SCC) from the head and neck, particularly in human papillomavirus-related carcinoma of Waldeyer's ring (4). However, cystic metastases should be separated from central necrosis following rapid tumor growth (5). Branchial cleft cyst carcinoma has long been a subject of discussion $(6,7)$.

This study investigated the data of patients who were admitted to the ENT Department by an ENT practitioner and diagnosed with a "branchial cleft cyst" requiring surgical treatment. The objective of the present study was to evaluate the significance of a cystic cervical mass in relation to etiology, diagnostic procedure and an algorithm for increased therapeutic efficiency.

\section{Patients and Methods}

This retrospective study reviewed the data of 133 consecutive patients who underwent removal of a cystic lateral neck lesion with or without neck dissection between 2006 and 2018 at one single center (Ruppiner University Hospital Neuruppin). Patients with apparent primary head and neck malignancy at clinical examination were excluded. Preoperatively, the patients underwent clinical examination. Sonography was performed to visualize the cystic formation. Additional computed tomography was performed if malignancy of the neck cyst was suspected.

In patients younger than 40 years, the neck mass was removed as an exclusive procedure. In patients who were 40 years or older, 
pan-endoscopy was performed to exclude malignant lesion of the upper aerodigestive tract at the beginning of the surgical procedure. After exclusion of a primary head and neck tumor by panendoscopy, patients with cystic neck lesions underwent tumor removal only if malignancy was not suspected macroscopically.

In patients older than 40 years with risk factors (e.g. smoking and alcohol abuse), intraoperative frozen section pathology was performed when suspected malignancy was found macroscopically. Suspected features of malignant neck cysts included tumors with a central cystic lesion surrounded by a variable thick capsule. Generally, the cut face of a cystic capsule appears grey and granular (5). When frozen section pathology demonstrated SCC, comprehensive neck dissection was performed in the same setting. Therefore, patients older than 40 years were informed preoperatively about the possible need for neck dissection. Neck surgery was regularly performed using a binocular loupe.

In patients with histologically confirmed SCC of the neck mass without proven primary tumor, we repeated the pan-endoscopy in a second setting and performed a bilateral tonsillectomy and took mapping biopsies of the tongue base and nasopharynx.

All investigations were performed according to accepted clinical practice and in compliance with the medical principles of the Declaration of Helsinki and German Federal Law. Informed consent was obtained from all patients prior to treatment. For this retrospective study, formal ethical approval was not required.

Statistical analysis was performed using IBM SPSS Statistics 23 (IBM Corp., Armonk, NY, USA). To compare patients' ages, the $t$-test for unpaired samples was used. Differences with $p$-values $\leq 0.05$ were regarded as significant.

\section{Results}

The study included data from 133 patients, 82 males and 51 females. The average size of the cystic lesions was $3 \mathrm{~cm}$ (range 1.5 to $8 \mathrm{~cm}$ ) using ultrasound examination. The current series included 69 left neck and 64 right neck biopsies. In cases with proven metastatic cancer, the side of metastases in the neck corresponded to the side of the primary tumor. Mean patient age was 44.1 years (range 5-91 years) and was significantly higher among patients with malignant diseases than with nonmalignant diseases ( 61.5 versus 36.3 years, $p=0.001)$. While malignancy was extremely rare in patients younger than 40 years (1.6\%), it increased significantly to $56 \%$ in patients $\geq 40$ years of age (Figure 1). While gender distribution among patients without malignancy was unremarkable (49 males, 43 females), the proportion of males with a malignant diagnosis was significantly higher (33 males, $80 \%, p=0.001$ ) (Figure 1).

In 92 out of $133(69 \%)$ cystic lesions, non-malignant disease was found, a branchial cleft cyst in the majority of cases $(85 \%)$. The mean age of this group was 33.5 (range 5 to 91) years. The second most important finding was cystic deformed lymph nodes carrying granulomatous infection (seven cases). Tuberculosis was proven in five cases. Table I indicates the histological diagnoses of the investigated patients with cystic lesions. In all but one case with nonmalignant findings, the removed tumor was used in the initial diagnosis.
Table I. Histological results ( $n=133)$.

\begin{tabular}{lc}
\hline & $\mathrm{N}(\%)$ \\
\hline Non-malignant cystic lesions & $92(100)$ \\
Branchial cleft cyst & $78(84.8)$ \\
Granulomatous infection & $7(7.6)$ \\
Tuberculosis & $5(5.4)$ \\
Epithelioid-cystic lymphadenitis & $2(2.2)$ \\
Warthin's tumor & $2(2.2)$ \\
Thyroid cyst & $1(1.1)$ \\
& \\
Malignant cystic lesions & $41(100)$ \\
Malignant lymphoma & $3(7.3)$ \\
Hodgkin's lymphoma & $1(2.4)$ \\
Non-Hodgkin's lymphoma & $2(4.9)$ \\
Carcinoma & $38(92.7)$ \\
Proven primary tumor & $23(56.1)$ \\
Secondary metastases (larynx, breast) & $2(4.2)$ \\
Primary manifestation & $21(51.2)$ \\
Upper aerodigestive tract & $19(46.3)$ \\
Tonsils & $10(24.4)$ \\
Nasopharynx & $4(9.6)$ \\
Base of the tongue & $2(4.9)$ \\
Hypopharynx & $3(7.3)$ \\
Parotid and thyroid gland & $2(4.9)$ \\
Primary tumor unknown & $15(36.6)$ \\
\hline
\end{tabular}

In 41 out of 133 (31\%) biopsies, malignant diseases were found which were located within a lymph node (Table I). SCC (35 cases, $85 \%$ ) was the most frequent histological entity. Three cases were diagnosed as malignant lymphoma. In all but one case, the primary tumors were located in the head and neck region. Waldeyer's ring carcinomas were the most significant. In further cases, the primary tumor of the cystic cervical lesion was found in the hypopharynx, the thyroid and parotid gland. Two other cases were diagnosed as late metastases from primary laryngeal and breast cancer tumors ( 3 and 9 years after initial therapy). In the current series, the neck lesion was the first manifestation of metastatic disease in 36 out of 38 cases, and in two out of three cases of malignant lymphoma. In 23 cases of proven metastatic disease, the cervical cystic lesion was the first and only clinical manifestation of primary or secondary carcinoma. In 15 cases it was not possible to detect a primary tumor. These cases were interpreted as metastases of cancer with unknown origin (CUP).

During the first surgical intervention, 66 out of 133 patients (50\%) underwent pan-endoscopy. The initial diagnosis of unknown primary tumor was made in 14 cases. In the remaining 52 cases, 40 frozen section evaluations were performed. In 16 cases, SCC was found and therefore neck dissection was performed in the same operation. In 24 cases, frozen section diagnosis was performed, which resulted in 18 non-malignant cases and six false-negative or not-evaluated 
100

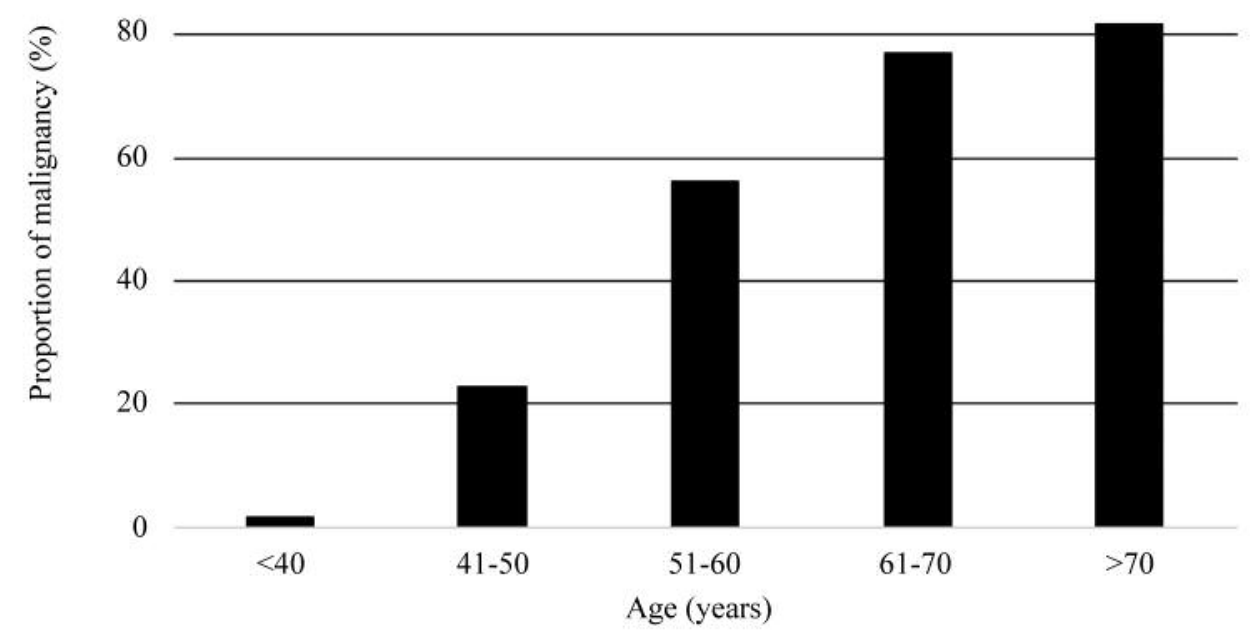

Cases, $\mathbf{n}$

Malignant

Non-malignant

All

$\begin{array}{rr}1 & 5 \\ 61 & 17 \\ 62 & 22\end{array}$

5

17

22
9

7

16

$\begin{array}{rr}17 & 9 \\ 5 & 2 \\ 22 & 11\end{array}$

Figure 1. Proportion of malignant findings according to age.

cases. A second setting to perform a neck dissection was required for three patients in the false-negative group and another three patients where no frozen section evaluation was performed after the definitive histological result reported SCC. In five cases, the primary tumor was found during a second pan-endoscopy after removal of the cystic mass (three by tonsillectomy, two by blind-biopsy from the nasopharynx) in cases with histologically confirmed SCC. One 16-year-old adolescent was diagnosed with cystic metastasis of a mucoepidermoid carcinoma and had neither pan-endoscopy nor intraoperative frozen section histology. Finally, 36 patients underwent at least one neck dissection (35 SCC, one mucoepidermoid carcinoma). In 30 out of 36 patients, neck dissection was performed during the first neck operation. Six patients underwent neck dissection in a second setting after removal of the cystic lesion.

HPV status was determined in 28 out of 35 cases diagnosed as SCC and proved positive in 10 cases (six tonsil cancer, five CUP, three cancer of the tongue base).

\section{Discussion}

Patients presenting with cystic neck lesions are relatively rare in the daily work of head and neck specialists. To increase therapeutic efficiency, a central challenge is to anticipate whether the mass represents a branchial cleft cyst or a malignant, particularly metastatic disease.

The two largest studies investigating cystic neck lesions include data from 130 patients and were monocentric (8) and multicentric (9). Further literature includes a review (5) and smaller series and case reports $(10,11)$. Other reports have different study designs (12). Therefore, this study presents the largest monocentric patient cohort to date of 133 consecutive patients who underwent surgery for a cystic mass. We discuss etiology, diagnostic aspects and clinical features, and present our algorithm to increase diagnostic and therapeutic efficiency.

Pathophysiological aspects. The majority of histological findings in patients presenting with a cystic neck lesion are non-malignant according to this study and the current literature $(8,9)$. The most frequent diagnosis $(59 \%)$ in the current study was branchial cleft cyst (Table I). In other series, frequencies of this entity up to $90 \%$ were reported (8-10). The second most frequent pathological result was cystic deformed lymph nodes caused by granulomatous infections with tuberculosis. This patient group included migrants from outer European countries younger than 40 years (13). Other non-malignant cystic neck lesions such as Warthin's tumor or thyroid cysts presented as individual cases consistent with the available literature $(9,10)$. 
The malignancy rate in the present study was $31 \%$ across all patients and significantly higher than in other investigations with rates between $9.2 \%$ and $14.4 \%(8,9,11,14)$. In patients older than 40 years, the percentage of malignant findings in the current study was $56 \%$. Other authors reported rates up to $31 \%(8,10,14)$. Malignant findings were extremely rare in patients under 40 years of age $(1.6 \%)$. On the other hand, nearly one-third (31/92) of the present cohort with nonmalignant findings were older than 40 years. Sira et al. (10) and Seven et al. (15) described a relevant number of papillary thyroid cancer metastases in patients younger than 40 years. In the current study, only one patient with thyroid cancer was found (female aged 79 years). The incidence of malignancy increases in patients older than 40 years $(2,3)$. The present results highlight the importance of patient age for differential diagnoses of cervical cystic lesions $(2,14)$. Grönlund et al. discuss a lower critical patient age of 35 years with respect to an increased incidence of HPV-related SCC (9).

The prevailing histological finding in the current study in patients with malignancy was SCC histology (85\%). The majority of primary tumors were located in Waldeyer's ring (8, 16). According to the current results, primary carcinoma of the hypopharynx should be included in the search for a primary tumor. Even malignant lymphoma may present as a cystic lesion. Cystic metastases from a primary tumor below the clavicle remain rare exceptions (17). In 15 patients with a histological diagnosis of SCC, no primary tumor was found, even after a meticulous search. These cases were considered as CUPs of an occult primary and were not regarded as branchial cleft cyst carcinoma $(18,19)$. In our opinion, we agree with former publications that branchial cleft cyst carcinomas either do not exist or are extremely rare $(6,7)$ (Table I).

Diagnostical aspects. A highly comprehensive history is necessary when patients present with a neck lesion. Anamneses should include infections of the upper aerodigestive tract with enlarged lymph nodes as well as symptoms and habits related to head and neck carcinoma (e.g. pain, dysphagia, smoking and drinking habits). The history should also include typical complaints of tuberculosis and sarcoidosis, and malignant lymphoma.

In the current series, the neck lesion was the first manifestation in most cases of non-malignant (91/92) and malignant (38/41) diseases.

The next steps are the ear, nose and throat examination. The majority of patients presented with a palpable, indolent and sometimes fluctuating cervical mass in level II of 3 to $6 \mathrm{~cm}$. None of our patients had suspicious findings on physical (endoscopic) examination, including flexible endoscopy. While every patient in our series underwent ultrasonography of the head and neck including the thyroid gland, $75 \%$ of adult patients older than 40 years were investigated additionally by computed tomographic (CT) or magnetic resonance imaging.
For superficial lesions, ultrasound is the first-level imaging study of choice because it is non-invasive, rapid, low-cost, and does not involve exposure to ionizing radiation (20). Using ultrasound to differentiate between non-malignant and malignant cystic lesions can be challenging. Misdiagnosis of malignant neck cysts may delay diagnosis (21). However, use of CT and magnetic resonance imaging may also have difficulties differentiating between non-malignant and malignant cystic lesions (22-24).

In a recent investigation, Abadi et al. identified 14 out of 20 patients with cancer in a retrospective series of 58 patients by positron-emission tomography/CT (25). However, none of our patient underwent such examination.

Fine-needle aspiration biopsy (FNAB) is frequently considered to be of paramount importance when diagnosing neck masses. Depending on personal experiences, FNAB has been associated with false-negative results in cases of malignant diseases when aspiration histology of cystic fluid was free of tumor cells. Discrepancies between FNAB and final histology, which cause time delay in searching for a primary tumor and the onset of therapy, are well documented in literature $(5,8,9,14)$. In our experience, FNAB delays diagnosis of malignant lymphoma. In patients with SCC with false-negative histological results, neck dissections are required in a second setting after definitive histology. Therefore, we do not recommend FNAB when diagnosing cystic neck lesions.

Consequently, our results support the opinion reported in other series that discrimination between a branchial cleft cyst and a malignancy is not possible based on history, clinical examination and radiological imaging including FNAB (5, $8,9)$. Histological examination is absolutely required. This algorithm increases therapeutic efficiency.

Therapeutic aspects. The large proportion of malignant findings in the current series emphasize the demand for early biopsy in patients with neck cysts to avoid delaying diagnosis and beginning therapy. Half of the patients (67/133) underwent resection of the neck mass without further procedures. In more than $20 \%$ (14/66) of the remaining patients, we found an occult primary tumor of the upper aerodigestive tract by pan-endoscopy. Palpation was of paramount importance when detecting carcinoma of Waldeyer's ring (11/14).

The remaining patients underwent open surgery. The primary objective of our approach is to avoid excision of a cyst before definitive therapy (neck dissection). We believe that the risk of complications and unnecessary radical surgery is increased if definitive surgery is secondary to resection of the neck cyst. Notwithstanding this, others have shown that neck surgery during a second setting does not necessarily result in a poorer outcome (26).

Intraoperative frozen section evaluation was performed in patients with suspected malignancy and without an 
endoscopically evident tumor in pan-endoscopy. Metastases of SCC were found in 16 out of 40 cases. In six cases, the frozen section was either false-negative or not evaluable (three cases each). According to our experience, intraoperative frozen section evaluation is a reliable method for ruling out malignant histology, e.g. SCC. As a result, neck dissection in a second setting could have been avoided in 30 out of 36 cases. The superior sensitivity of frozen section evaluation in cystic neck lesions compared with FNAB has been demonstrated by Begbie et al. (27).

Moreover, our results outline the need for a second panendoscopy with tonsillectomy and mapping biopsies of the tongue base and nasopharynx. In the current study, we performed this procedure after negative pan-endoscopy and proven SCC in the cervical neck cyst. Thereby, five unknown primary tumors were detected.

Patients with metastatic head and neck cancer received postoperative radio- or radiochemotherapy through our practice. When the primary tumor remained occult, additional mucosal dosing of the upper aerodigestive tract excluding the oral cavity was performed (3).

Prognostic aspects. In the current series, HPV status was determined in $50 \%$ of those with tonsil cancer and $27 \%$ of those with CUP. The association between tumorigenic HPV and cystic morphology of metastasis has been discussed (4). HPV positivity in cancer of Waldeyer's ring, especially tonsillar cancer, has been demonstrated by several authors, while the majority of branchial cleft cysts were proven HPVnegative $(8,28)$. Therefore, the present results confirm findings of previous reports $(24,29,30)$.

\section{Conclusion}

According to our results, malignancy is the prevailing finding in patients older than 40 years who present with cystic lesions of the lateral neck. We recommend an algorithm including pan-endoscopy, evaluation of intraoperative frozen section of the cystic mass and possible neck dissection in order to avoid invasive neck surgery before definitive surgical neck therapy and treatment delays. A second pan-endoscopy with tonsillectomy and random biopsies are required in patients with unexpected SCC in the definitive histological result.

\section{Conflicts of Interest}

None.

\section{Authors' Contributions}

Achim Franzen: Designed the study, collected and analyzed data. Thomas Günzel: Wrote the article. Andre Buchali: Wrote the article. Annekatrin Coordes: Collected and analyzed data, wrote the article.

\section{References}

1 Al-Khateeb TH and Al Zoubi F: Congenital neck masses: A descriptive retrospective study of 252 cases. J Oral Maxillofac Surg 65(11): 2242-2247, 2007. PMID: 17954320. DOI: 10.1016/ j.joms.2006.11.039

2 Luna MA and Pfaltz M: Cysts of the neck, unknown primary and neck dissection. In: Diagnostic Surgical Pathology of the Head and Neck. Gnepp D (ed.). Saunders: Philadelphia, pp. 671, 2000.

3 Franzen AM, Lieder A and Buchali A: Significance of secondary mucosal primaries in patients with a cervical cup-syndrome. Laryngorhinootologie 95(7): 477-481, 2016. PMID: 27172108. DOI: $10.1055 / \mathrm{s}-0042-100907$

4 Goldenberg D, Begum S, Westra WH, Khan Z, Sciubba J, Pai SI, Califano JA, Tufano RP and Koch WM: Cystic lymph node metastasis in patients with head and neck cancer: An HPVassociated phenomenon. Head Neck 30(7): 898-903, 2008. PMID: 18383529. DOI: 10.1002/hed.20796

5 Goldenberg D, Sciubba J and Koch WM: Cystic metastasis from head and neck squamous cell cancer: A distinct disease variant? Head Neck 28(7): 633-638, 2006. PMID: 16477605. DOI: 10.1002/hed.20381

6 Briggs RD, Pou AM and Schnadig VJ: Cystic metastasis versus branchial cleft carcinoma: A diagnostic challenge. Laryngoscope 112(6): 1010-1014, 2002. PMID: 12160265. DOI: 10.1097/00 005537-200206000-00014

7 Bradley PT and Bradley PJ: Branchial cleft cyst carcinoma: Fact or fiction? Curr Opin Otolaryngol Head Neck Surg 21(2): 118-123, 2013. PMID: 23328541. DOI: 10.1097/MOO.0b013e328 35cebde

8 Koch EM, Fazel A and Hoffmann M: Cystic masses of the lateral neck - proposition of an algorithm for increased treatment efficiency. J Craniomaxillofac Surg 46(9): 1664-1668, 2018. PMID: 29983308. DOI: 10.1016/j.jcms.2018.06.004

9 Gronlund S, Mey K, Andersen E and Rasmussen ER: The true malignancy rate in 135 patients with preoperative diagnosis of a lateral neck cyst. Laryngoscope Investig Otolaryngol 1(4): 7882, 2016. PMID: 28894805. DOI: 10.1002/lio2.23

10 Sira J and Makura ZG: Differential diagnosis of cystic neck lesions. Ann Otol Rhinol Laryngol 120(6): 409-413, 2011. PMID: 21774450. DOI: 10.1177/000348941112000611

11 Yehuda M, Schechter ME, Abu-Ghanem N, Golan G, Horowitz G, Fliss DM and Abu-Ghanem S: The incidence of malignancy in clinically benign cystic lesions of the lateral neck: Our experience and proposed diagnostic algorithm. Eur Arch Otorhinolaryngol 275(3): 767-773, 2018. PMID: 29282522. DOI: $10.1007 / \mathrm{s} 00405-017-4855-6$

12 Gourin A, Garzon AA, Rosen Y and Lyons HA: Bronchogenic cysts. Broad spectrum of presentation. NY State J Med 76(5): 714-719, 1976. PMID: 1063319.

13 Deveci HS, Kule M, Kule ZA and Habesoglu TE: Diagnostic challenges in cervical tuberculous lymphadenitis: A review. North Clin Istanb 3(2): 150-155, 2016. PMID: 28058405. DOI: 10.14744/nci.2016.20982

14 Gourin CG and Johnson JT: Incidence of unsuspected metastases in lateral cervical cysts. Laryngoscope 110(10 Pt 1): 1637-1641, 2000. PMID: 11037817. DOI: 10.1097/00005537-20001000000012

15 Seven H, Gurkan A, Cinar U, Vural C and Turgut S: Incidence of occult thyroid carcinoma metastases in lateral cervical cysts. Am J Otolaryngol 25(1): 11-17, 2004. PMID: 15011201. 
16 Svajdler M Jr., Kaspirkova J, Hadravsky L, Laco J, Dubinsky P, Straka L, Ondic O, Michal M and Skalova A: Origin of cystic squamous cell carcinoma metastases in head and neck lymph nodes: Addition of EBV testing improves diagnostic accuracy. Pathol Res Pract 212(6): 524-531, 2016. PMID: 27013059. DOI: 10.1016/j.prp.2016.03.002

17 Franzen A, Gunzel T, Buchali A and Coordes A: Etiologic and differential diagnostic significance of tumor location in the supraclavicular fossa. Laryngoscope 128(3): 646-650, 2018. PMID: 28727141. DOI: 10.1002/lary.26775

18 Tribius S, Hoffmann AS, Bastrop S, Gorogh T, Haag J, Rocken C, Clauditz T, Grob T, Wilczak W, Tennstedt P, Borcherding A, Petersen $\mathrm{C}$ and Hoffmann M: HPV status in patients with head and neck of carcinoma of unknown primary site: HPV, tobacco smoking, and outcome. Oral Oncol 48(11): 1178-1184, 2012. PMID: 22739067. DOI: 10.1016/j.oraloncology.2012.05.022

19 Strojan P, Ferlito A, Medina JE, Woolgar JA, Rinaldo A, Robbins KT, Fagan JJ, Mendenhall WM, Paleri V, Silver CE, Olsen KD, Corry J, Suarez C, Rodrigo JP, Langendijk JA, Devaney KO, Kowalski LP, Hartl DM, Haigentz M Jr., Werner JA, Pellitteri PK, de Bree R, Wolf GT, Takes RP, Genden EM, Hinni ML, Mondin $\mathrm{V}$, Shaha AR and Barnes L: Contemporary management of lymph node metastases from an unknown primary to the neck: I. A review of diagnostic approaches. Head Neck 35(1): 123-132, 2013. PMID: 22034046. DOI: 10.1002/hed.21898

20 Valentino M, Quiligotti C and Carone L: Branchial cleft cyst. J Ultrasound 16(1): 17-20, 2013. PMID: 24046795. DOI: 10.1007/ s40477-013-0004-2

21 Goyal N, Zacharia TT and Goldenberg D: Differentiation of branchial cleft cysts and malignant cystic adenopathy of pharyngeal origin. Am J Roentgenol 199(2): W216-221, 2012. PMID: 22826424. DOI: 10.2214/AJR.11.8120

22 Lindberg R: Distribution of cervical lymph node metastases from squamous cell carcinoma of the upper respiratory and digestive tracts. Cancer 29(6): 1446-1449, 1972. PMID: 503123 8 DOI: 10.1002/1097-0142(197206)29:6<1446::aid-cncr282029 0604>3.0.co;2-c

23 Ellison E, LaPuerta P and Martin SE: Supraclavicular masses: Results of a series of 309 cases biopsied by fine needle aspiration. Head Neck 21(3): 239-246, 1999. PMID: 10208667.

24 Aldridge T, Kusanale A, Colbert S and Brennan PA: Supraclavicular metastases from distant primaries: What is the role of the head and neck surgeon? Br J Oral Maxillofac Surg 51(4): 288-293, 2013. PMID: 22591766. DOI: 10.1016/j.bjoms. 2012.03.016
25 Abadi P, Johansen A, Godballe C, Gerke O, Hoilund-Carlsen PF and Thomassen A: (18)F-FDG PET/CT to differentiate malignant necrotic lymph node from benign cystic lesions in the neck. Ann Nucl Med 31(2): 101-108, 2017. PMID: 27921286. DOI: $10.1007 / \mathrm{s} 12149-016-1142-3$

26 Zenga J, Graboyes EM, Haughey BH, Paniello RC, Mehrad M, Lewis JS Jr., Thorstad WL, Nussenbaum B and Rich JT: Definitive surgical therapy after open neck biopsy for HPVrelated oropharyngeal cancer. Otolaryngol Head Neck Surg 154(4): 657-666, 2016. PMID: 26861231. DOI: 10.1177/ 0194599815627642

27 Begbie F, Visvanathan V and Clark LJ: Fine-needle aspiration cytology versus frozen section in branchial cleft cysts. J Laryngol Otol 129(2): 174-178, 2015. PMID: 25684337. DOI: $10.1017 /$ S0022215115000067

28 Sivars L, Landin D, Haeggblom L, Tertipis N, Grun N, Bersani C, Marklund L, Ghaderi M, Nasman A, Ramqvist T, Nordfors C, Munck-Wikland E, Tani E and Dalianis T: Human papillomavirus DNA detection in fine-needle aspirates as indicator of human papillomavirus-positive oropharyngeal squamous cell carcinoma: A prospective study. Head Neck 39(3): 419-426, 2017. PMID: 27898186. DOI: 10.1002/hed.24641

29 Fakhry C and Gillison ML: Clinical implications of human papillomavirus in head and neck cancers. Clin Oncol 24: 26062611, 2006. PMID: 16763272. DOI: 10.1200/JCO.2006.06.1291

30 Huang YH, Yeh CH, Cheng NM, Lin CY, Wang HM, Ko SF, Toh $\mathrm{CH}$, Yen TC, Liao CT and Ng SH: Cystic nodal metastasis in patients with oropharyngeal squamous cell carcinoma receiving chemoradiotherapy: Relationship with human papillomavirus status and failure patterns. PLoS One 7: 7-12, 2017. PMID: 28686646. DOI: 10.1371/journal.pone.0180779 